RESEARCH PAPER RP1056

Part of Journal of Research of the National Bureau of Standards, Volume 19, December 1937

\title{
EFFECT OF YAW ON VANE ANEMOMETERS
}

\author{
By Roy H. Heald and Paul S. Ballif
}

\section{ABSTRACT}

The effect of yaw on the performance of three vane-type anemometers was determined in the wind tunnel. Observations were made for angles of yaw within a range of 35 degrees on either side of the zero position. Maximum positive errors of 1,4 , and 5.5 percent were found for the three instruments when the angle of yaw was approximately 15 degrees. The magnitude of the error depends on the design.

\section{CONTENTS}

I. Introduction

II. Description of the anemometers

III. Method of test

IV. Results _.

V. Conclusions

\section{INTRODUCTION}

The customary practice in using a vane anemometer is to mount the instrument so that the axis of rotation of the vane wheel is parallel to the axis of the fan or duct for which measurements are to be made. Usually no attempt is made to determine whether or not the air flow is parallel to the duct or fan axis, it being assumed that no appreciable error is introduced on this account. Ower ${ }^{1}$ states, for a particular instrument, that the directional effects due to yaw may introduce errors of the order of 1 percent for angles of inclination less than about 20 degrees, but points out the uncertainties involved in drawing conclusions from the performance of a single instrument. Differences in design of anemometers constructed by different manufacturers are often encountered in practice. Hence differences in response to directional effects are to be expected, especially when the direction of air flow makes a large angle with the reference axis.

Recently, in determining the air deliveries of electric fans, the direction of air flow was found to be inclined 20 degrees or more to any plane containing the fan axis. In such cases, accurate results can be obtained with an anemometer mounted with its axis parallel to the fan axis only if the correction factor for airstream inclination is known and applied. For this reason an investigation was undertaken

\footnotetext{
1 E. Ower. Measurement of Air Flow, 2d ed., p. 119. (Chapman and Hall, London, 1933.)
} 
to determine the effect of airstream inclination, in yaw, ${ }^{2}$ on the performance of the three vane anemometers used in the fan tests. The information may be of interest to other investigators who have occasion to use vane anemometers under unfavorable conditions of air flow.

\section{DESCRIPTION OF THE ANEMOMETERS}

The anemometers used in these experiments are shown in the halftone figure 1, and in the detail drawings, figure 2. The length/ diameter ratios of the cylindrical housings are 0.43 for anemometer $A ; 0.32$ for anemometer $B$; and 0.59 for anemometer $C$. Anemometers

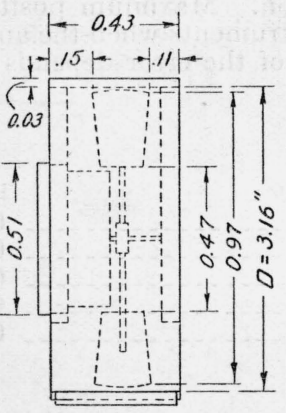

$A$

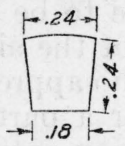

Blade dimensions (Plan)

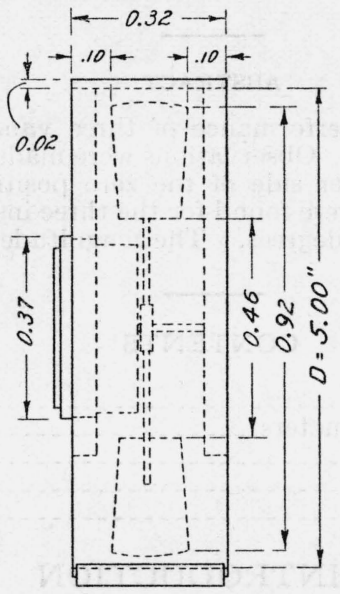

$B$

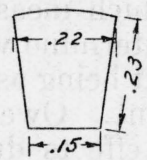

Blade dimensions (Plan)

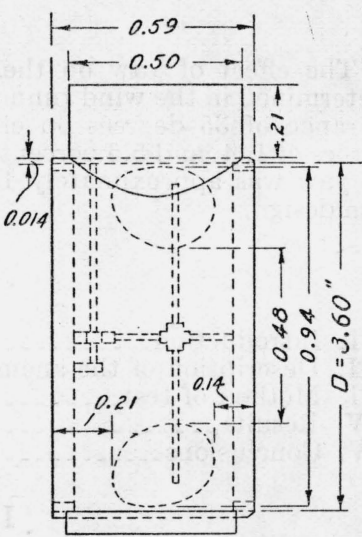

C

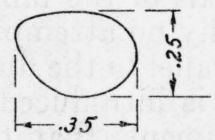

Blade dimensions (Plan)

Figure 2.-Line drawings of the anemometers.

The dimensions are given as decimals of the inside diameter of the housing.

$A$ and $B$ are dial indicating. Anemometer $C$ transmits signals electrically.

The plane of rotation of the upstream tips of the rotor vanes is 0.11 diameter within the housing for anemometer $A ; 0.10$ diameter for anemometer $B$; and 0.14 diameter for anemometer $C$, the inside diameter of each housing being used as a reference. The rotor vanes have plane surfaces.

${ }_{2}^{2}$ The yaw angle is defined as the angle in a horizontal plane between the axis of the instrument and the direction of air flow. Since the anemometer is nearly a symmetrical instrument the effects of pitch, i. e., of inclination in a plane normal to the yaw plane, are not expected to differ greatly from the effects of yaw. 


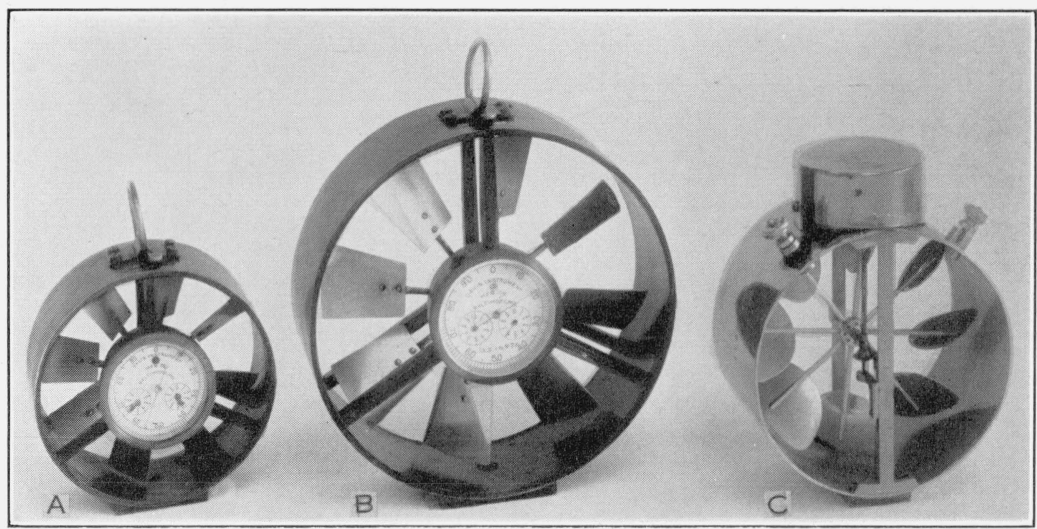

Figure 1. - Three vane-type anemometers $(A, B$, and $C)$ used in the experiments. 


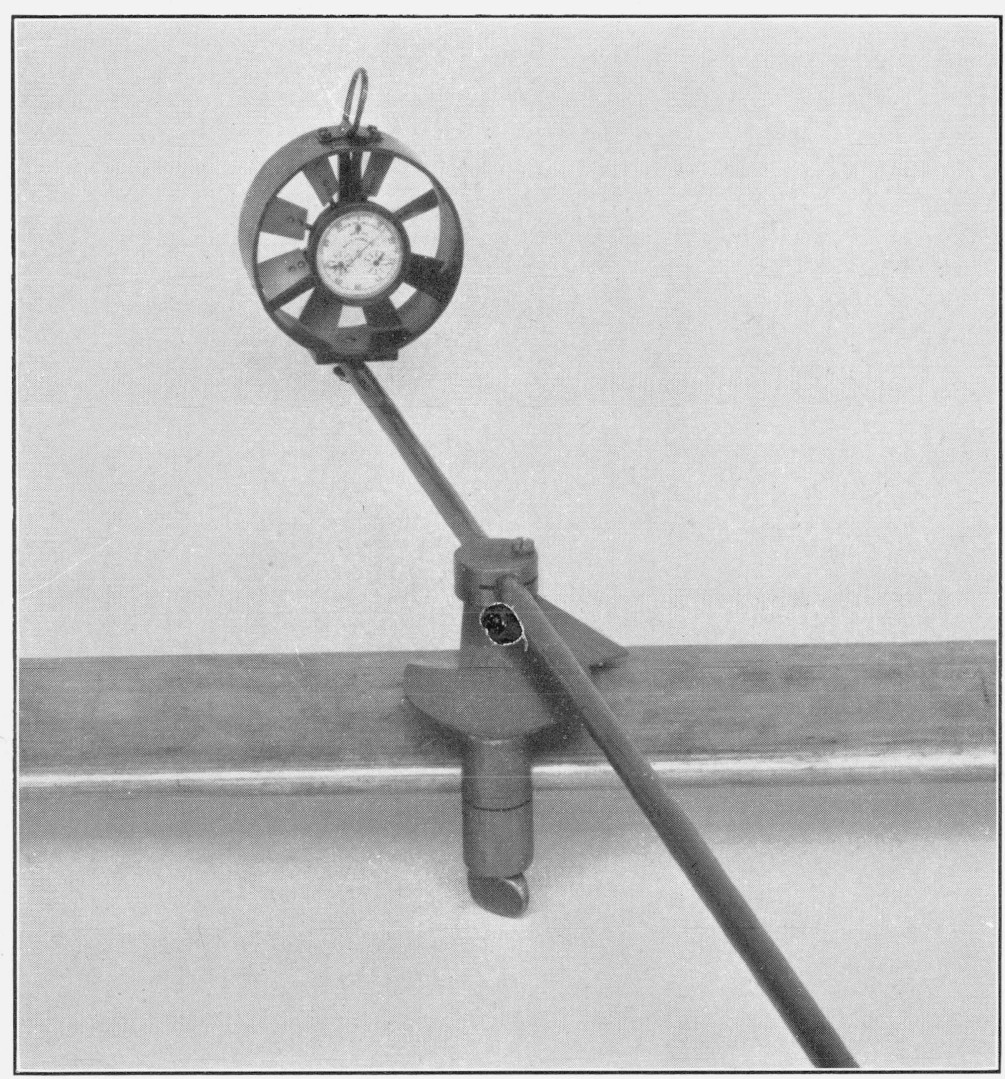

Figure 3.-Anemometer support. 


\section{METHOD OF TEST}

The anemometers were tested in the 54-inch wind tunnel of the National Bureau of Standards. The instrument under test was attached to a cylindrical mounting rod $3 / 8$ inch in diameter and 3 feet long. The mounting rod was clamped to a faired strut (fig. 3) fixed to the tunnel walls. The effect of the presence of the strut and clamping device was determined for each anemometer by observing the indicated velocity when the instrument was mounted various distances ahead of the strut. As may be seen in figure 4, the effect of the strut and clamp was found to be small for positions more than 6 inches ahead of the strut.

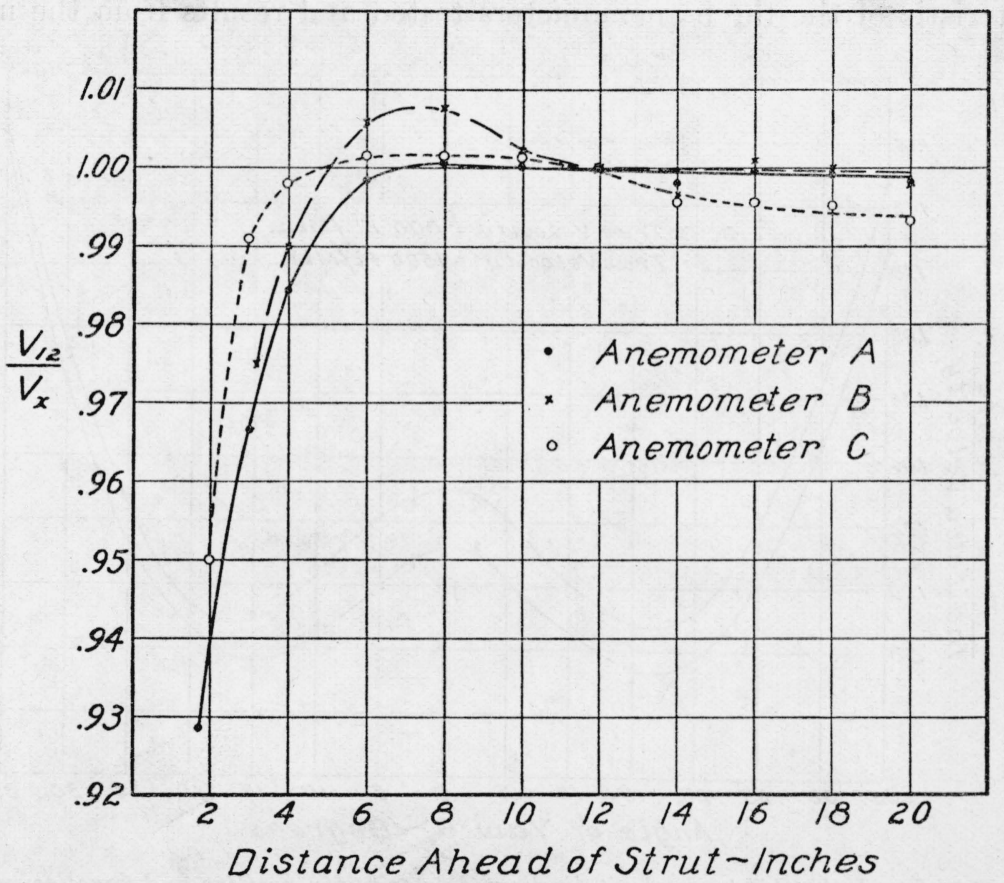

FIgURe 4.-Effect of support interference on the velocity indications for zero yaw.

$V_{12}=$ indicated velocity at point 12 inches ahead of the strut.

$V_{x}=$ indicated velocity at point $x$ inches ahead of the strut.

The method of mounting the anemometers, illustrated in figure 3, permitted yawing of the mounting rod through an angle of about 45 degrees on either side of the center position. Yaw settings were read on a graduated scale mounted on the clamp. The mounting screw in the base of the anemometer was maintained at a point 12 inches ahead of the strut and midway between opposite walls of the wind tunnel for all angle settings by adjusting the clamp and mounting rod. Thus the effect of the small velocity gradient in the wind tunnel was eliminated.

The velocity indicated by each anemometer was observed for angles of yaw in intervals of 5 degrees through a range of -35 to +35 degrees. At least two observations of indicated velocity were made 
for each yaw setting and for each of two air velocities, approximately 800 feet per minute and approximately 1,500 feet per minute. The constancy of the air velocity through the tunnel during the tests was of the order of 1 percent. The time interval for each observation was about 2 minutes.

\section{RESULTS}

The ratio of the true air velocity in the wind tunnel to the velocity indicated by the anemometer was computed for each observation. The results for anemometer $B$ are plotted in figure 5 . The curves are similar to those obtained for instruments $A$ and $C$. The difference in the value of the ratio, true velocity/indicated velocity, for air velocities of 800 feet per minute and 1,500 feet per minute is characteristic of the three anemometers tested and results from the non-

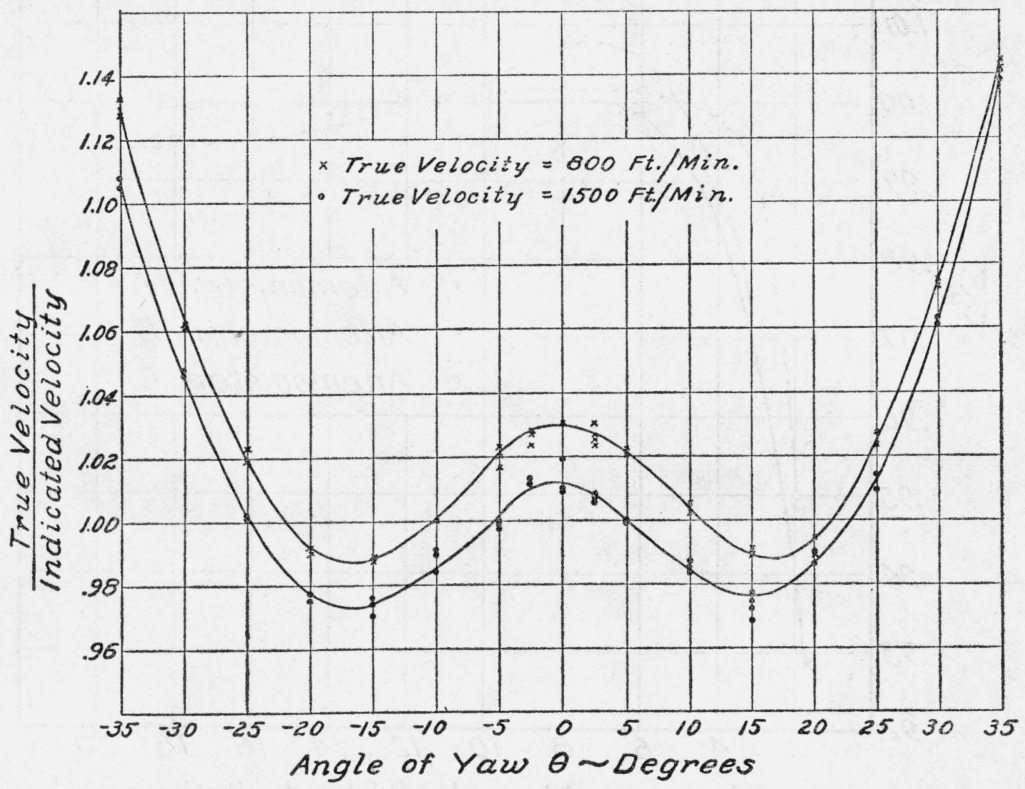

FIGURE 5.-Values of true velocity/indicated velocity for positive and negative angles of yaw, anemometer $B$.

linear relationship between the friction of the bearings and indicatingmechanism load and the torque applied to the vanes by the air stream. Ordinarily, corrections for this difference are made by using the calibration curve for the instrument.

The ratio of the indicated velocity $V_{0}$ for zero yaw, to the indicated velocity $V_{\theta}$ for angle of yaw $\theta$, was computed for each observation. The curves in figure 6 show the mean values of $V_{0} / V_{\theta}$, all of the observations obtained for a given angle of yaw for each instrument being used. In general, the indicated velocity increased as the angle of yaw was increased, reaching a maximum when the angle of yaw was about 15 degrees. Further increase in the angle of yaw resulted in a decrease in indicated velocity. The indicated velocity for 15 degrees of yaw, for anemometers $A, B$, and $C$, was about $5.5,4$, and 
1 percent, respectively, greater than the indicated velocity at zero yaw; for approximately 30,27 , and 18 degrees of yaw, respectively, the indicated velocity was the same as for zero yaw and for greater angles of yaw the indicated velocity was less than for zero yaw.

It will be noted from figures 1 and 2 that anemometer $A$ which showed a maximum positive error of 5.5 percent, is substantially different in design from anemometer $C$, which showed a maximum positive error of about 1 percent. For example, there is a decided difference in the shape of the vanes. Also, the dial of anemometer $A$ exerts a blocking effect over about 25 percent of the area of the housing, while the housing of anemometer $C$ is comparatively free from obstruction. Further, the length/diameter ratios of the housings are

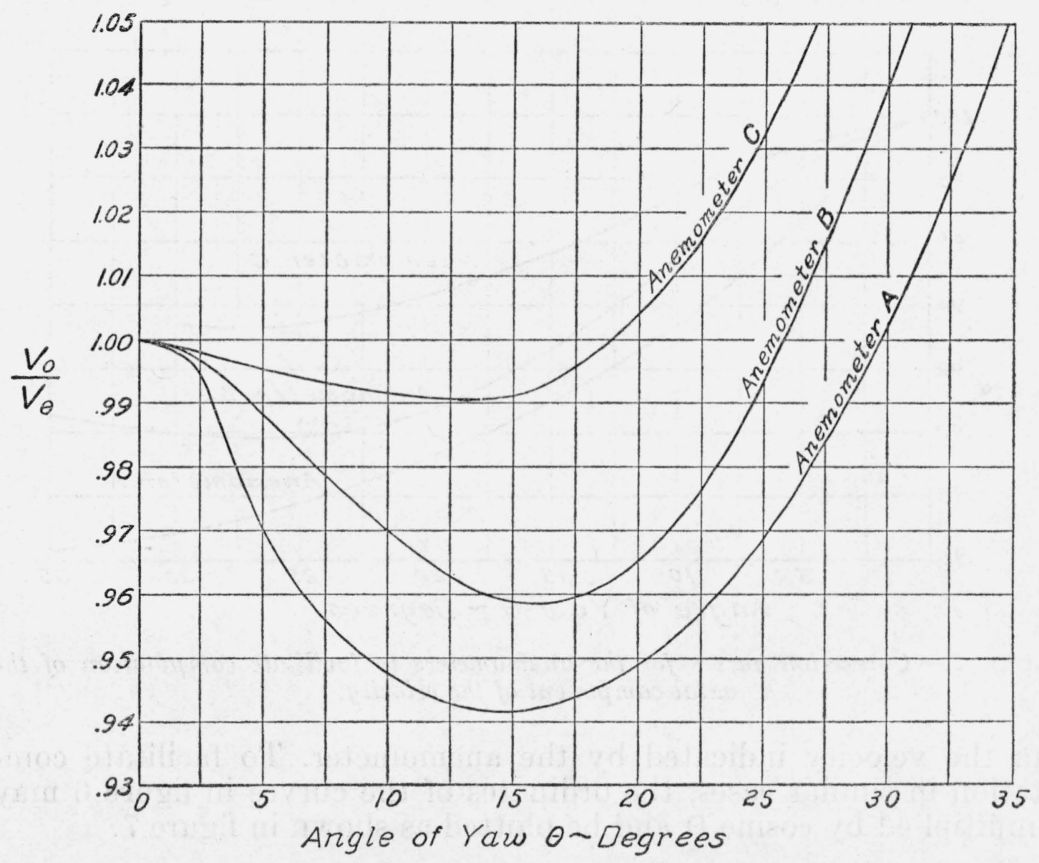

FIGURE 6.-Effect of angle of yaw on the velocity indications.

The curves give mean values of $V_{0} / V_{\theta}$.

different. Other dissimilarities are to be found, such as differences in wall thickness and external fittings.

A few experiments were made wherein the length/diameter ratio of anemometer $A$ was increased about one-third by adding a cylindrical extension to the housing. The results showed a decrease of a small amount in the directional error. Hence it appears that the magnitude of the error due to inclination of the airstream depends on the design of the anemometer, and it is not possible to ascribe the differences in directional sensitivity of the instruments used to any one design characteristic.

The use of the curves in figure 6 is shown by the following example. Suppose the reading of anemometer $A$, corrected for the calibration error at zero yaw, to be 1,240 feet per minute. Also suppose the 
direction of air flow with respect to the anemometer axis to be 20 degrees. This angle corresponds to the angle $\theta$ and usually may be determined with sufficient accuracy by means of a light thread and a protractor. Referring to figure 6, the correction factor for anemometer $A$ when $\theta$ is 20 degrees is 0.949 . Hence the true velocity, in a direction making an angle of 20 degrees with the anemometer axis, is $1,240 \times 0.949=1,176$ feet per minute.

In most cases it is desired to know the component of the airstream velocity parallel to the axis of the fan or duct. This component is determined by resolving the true velocity, obtained as above, in the direction of the anemometer axis. In the example the value is $1,176 \times$ $\cos 20^{\circ}=1,105$ feet per minute. It will be noted that the component of airstream velocity parallel to the axis of the fan is 10.8 percent less

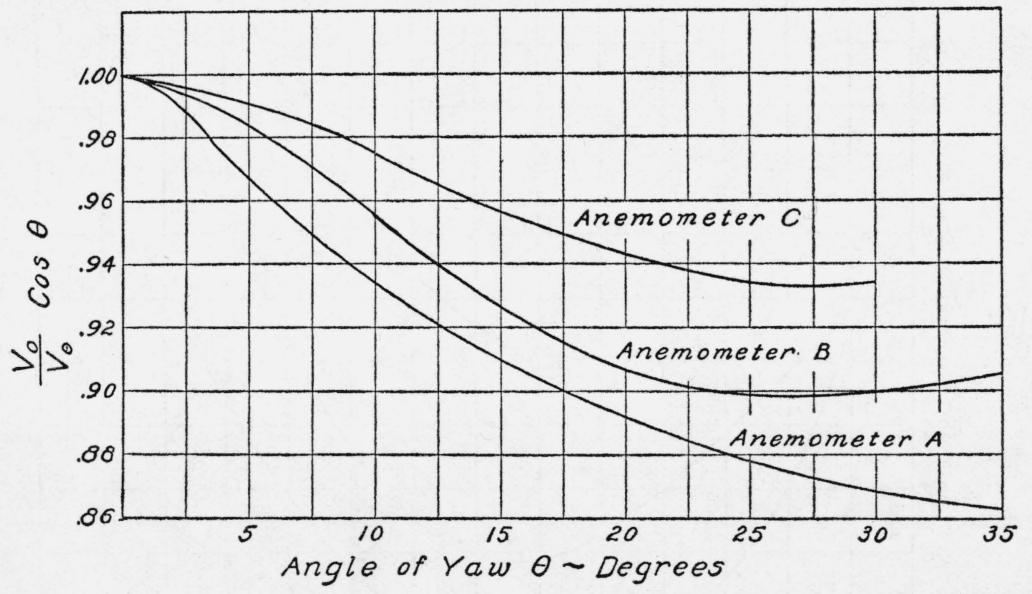

FIGURE 7.-Correction curves for the anemometers to facilitate computation of the axial component of the velocity.

than the velocity indicated by the anemometer. To facilitate computation in similar cases, the ordinates of the curves in figure 6 may be multiplied by cosine $\theta$ and be plotted as shown in figure 7 .

\section{CONCLUSIONS}

The effect of airstream inclination on the performance of three vane anemometers was determined in the wind tunnel. The results show the presence of substantial directional errors within the 0- to 35degree range of yaw angles. A maximum directional error of about 5.5 percent occurred when one of the instruments was displaced about 15 degrees from alignment with the air stream of the wind tunnel. An error of about 1 percent was observed when an anemometer of substantially the same diameter but of different design was displaced the same amount. The experiments indicate that the magnitude of the error due to inclination of the air stream depends on the design of the anemometer.

Washington, September 25, 1937. 